\title{
Fabrication of graphene: CdSe quantum dots/CdS nanorod heterojunction photodetector and role of graphene to enhance the photoresponsive characteristics
}

Chandrasekar Perumal Veeramalai ( $\sim$ pv_chandrasekar@yahoo.com )

Minzu University of China

Pratap kollu

University of Hyderabad

Guochen Lin

Minzu University of China

Xiaoming Zhang

Minzu University of China

Chuanbo Li

Minzu University of China

\section{Research Article}

Keywords: CdSe QDs, graphene, CdS nanorods, heterojunction, photodetector

Posted Date: February 11th, 2021

DOI: https://doi.org/10.21203/rs.3.rs-231208/v1

License: (c) (1) This work is licensed under a Creative Commons Attribution 4.0 International License. Read Full License

Version of Record: A version of this preprint was published at Nanotechnology on May 10th, 2021. See the published version at https://doi.org/10.1088/1361-6528/abf87a. 


\section{Fabrication of graphene: CdSe quantum dots/CdS nanorod heterojunction photodetector and role of graphene to enhance the photoresponsive characteristics}

Chandrasekar Perumal Veeramalai*a, Pratap kollu ${ }^{b}$, Guochen Lin ${ }^{a}$, Xiaoming Zhang ${ }^{a}$, Chuanbo $L i * a, c$

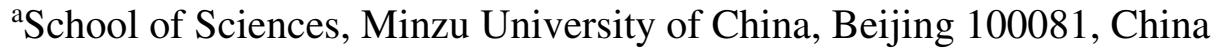

${ }^{\mathrm{b}}$ CASEST, School of Physics, University of Hyderabad, Prof. C.R. Rao Road, Gachibowli, Hyderabad -500046, India

${ }^{c}$ Optoelectronics Research Center, Minzu University of China, Beijing 100081, China E-mail: Corresponding author: cbli@ muc.edu.cn,

pv_chandrasekar@yahoo.com

Integration of graphene with semiconducting quantum dots (QDs) provides an elegant way to access the intrinsic properties of graphene and optical properties of QDs in a single hand to realize the high-performance optoelectronic devices. In the present study, high-performance photodetector based on graphene: CdSe QDs/CdS nanorod heterostructures, are demonstrated. The resulting heterojunction photodetector with device configuration ITO/graphene: $\mathrm{CdSe} / \mathrm{CdS}$ nanorods/Ag show excellent operating characteristics including a maximum photoresponsivity of $15.95 \mathrm{AW}^{-1}$ and specific detectivity of $6.85 \times 10^{12}$ Jones measured at $530 \mathrm{~nm}$. The device exhibits a photoresponse rise time of $545 \mathrm{~ms}$ and a decay time of $539 \mathrm{~ms}$. Furthermore, the effect of graphene nanosheets on the performance enhancement of heterojunction photodetector was explored. The results indicate that, due to the enhanced energy transfer from photoexcited QDs to graphene layer, light absorption is increased and excitons are generated. Also, the graphene: CdSe QDs/CdS nanorod interface can facilitate charge carrier transport effectively. This work provides a promising approach to develop high-performance visible-light photodetectors and utilization of advantageous features of graphene in optoelectronic devices.

Keywords: CdSe QDs; graphene; CdS nanorods; heterojunction; photodetector 


\section{Introduction}

Graphene nanosheets, a honeycomb structured carbon material, possess unique properties like high thermal stability, mechanical strength, and excellent electrical conductivity[1,2]. By virtue of its distinct characteristics, attention was drawn to represent graphene in the development of novel devices which can be used in various applications spanned from electronics to the medical field[3-5]. However, the zero-band gap nature of graphene restricts its application in optoelectronic devices effectively. This obstacle could be overcome by the modification of graphene surface with metals, metal oxides, quantum dots, and will further broaden the application field with tunable properties[6-8].

Meanwhile, semiconductor QDs are zero-dimensional materials exhibits unique optical and electrical properties due to their small size of $2-10 \mathrm{~nm}[9,10]$. With the inherent fascinating properties, semiconducting QD has potential applications in photodetectors, solar cells, bioimaging, and many more[11-13]. Nevertheless, graphene and semiconducting QDs also have their disadvantages. For instance, the de-coherence and low carrier mobilities limit the optical gain of QD materials. And also, the low light absorption, ultra-fast recombination of photogenerated carriers, and ease of aggregation in solution restrict the graphene application in optoelectronic devices[14]. Therefore, in recent years, there has been renewed interest in the integration of graphene with nanoparticles. In the graphene nanocomposite structure, the semiconducting nanoparticles act as a light-absorbing layer to produce electron-hole pairs, and then, charge carriers are rapidly transferred through high mobility graphene layers[15]. Moreover, it is possible to extend the QDs emission to longer wavelengths by combining the electronic properties of graphene with those of QDs in graphene: QD nanocomposites [16]. One of the important issues in the attachment of QDs on the surface of graphene is the monodispersity, which is essential for controlled charge transfer across the graphene layers. 
Therefore, many efforts were devoted to synthesizing graphene-QD hybrid structures and designing photoelectric devices. For example, Geng et al. demonstrated the non-covalent attachment of CdSe QDs to graphene to realize highly transparent semiconducting films[17]. Guo et al have developed a strategy to fabricate a solar cell using CdSe QDs-graphene and achieved the incident photon-to-charge-carrier conversion efficiency (IPCE) of 16\%, a large improvement from the graphene-only and QD-only devices (IPCE<6\%)[15]. The decoration of CdSe QDs on graphene sheets and the effect of graphene inclusion on optoelectronic properties of the QD based device was investigated by Kim et.al[18]. Sun et al. have reported the CVD-grown monolayer graphene-PbS QDs based flexible infrared photodetector on plastic substrates and the device has shown enhanced photoresponsivity of $10^{7} \mathrm{AW}^{-1}[19]$. Recently, Chen et al. proposed a photodetector of intercalated graphene layers with thick $\mathrm{PbS}$ QDs films (i.e. alternating layers of QDs and graphene) and achieved the efficient charge collection over the spectral range from visible to IR region[20]. by adopting the advanced technique of ink-jet printing, cook et al. have fabricated $\mathrm{ZnO/graphene} \mathrm{nanoplatelet} \mathrm{bulk} \mathrm{heterojunction} \mathrm{UV} \mathrm{photodetector} \mathrm{and} \mathrm{its} \mathrm{photoresponsivity} \mathrm{value}$ reaches up to $2.2 \mathrm{AW}^{-1}[21]$. Furthermore, charge carrier dynamics of nanoparticle (quantum dots)/graphene nanocomposites also have been studied experimentally and theoretically[22-25]. Specifically, the photoluminescence of CdSe QDs in graphene nanohybrids is reduced both in intensity and lifetime due to strong interaction and energy transfer[26]. These above-mentioned works highlight the importance of graphene's role to enhance the charge carrier dissociation and transport in the optoelectronic device. However, to the best of our knowledge, only a little research is reported on the effect of graphene in heterojunction photodetectors. For instance, Konstantatos et al. demonstrated monolayer or bilayer graphene-PbS QDs heterojunction phototransistor, where trapped charges at the interface causing a photogate effect and enhance the device 
photoresponsivity up to $10^{7} \mathrm{AW}^{-1}$ which is significantly higher than graphene only device of $10^{-2}$ $\mathrm{AW}^{-1}[27]$. Therefore, there is an immediate need for studying the optoelectronic properties of graphene: QD nanocomposites and specifically, the role of graphene on performance metrics of optoelectronic devices.

Herein, we present the hybrid photodetector consists of graphene: CdSe QDs and CdS nanorod. The graphene: CdSe QDs nanocomposite was synthesized via low-temperature in-situ synthesis technique. The PL and TRPL studies of graphene: CdSe QDs nanocomposite showed an obvious quenching effect compared to the pure CdSe QDs as the reason for fast separation and transfer of photo-induced charge carriers between CdSe QDs and graphene layers. The asfabricated hybrid heterojunction photodetector with device structure ITO/graphene: CdSe QDs $/ \mathrm{CdS} / \mathrm{Ag}$ shows enhanced photo responsivity of $15.95 \mathrm{AW}^{-1}$ and specific detectivity of $6.85 \times 10^{12}$ Jones which is significantly higher than ITO/CdSe/CdS/Ag device. Our work demonstrates the great potential of graphene: QD nanocomposite as the photoactive layer in the high-performance photodetector.

\section{Experimental methods}

2.1 Materials: All reagents were the technical grade of high purity, is used without further purification.1-Octadecene $\left(\mathrm{C}_{16} \mathrm{H}_{36}\right.$, purity $\left.99.0 \%\right)$ and Trioctylphosphine $\left(\mathrm{C}_{24} \mathrm{H}_{51} \mathrm{P}\right.$, purity $>95.0 \%$ ), were purchased from Aladdin Industrial Corporation, Shanghai, P.R.China. nOctylamine $\left(\mathrm{C}_{8} \mathrm{H}_{19} \mathrm{~N}\right.$, purity $\left.>99.0 \%\right)$, Selenium powder (Se, purity $\left.~ 99.0 \%\right)$, Cadmium acetate dihydrate $\left(\mathrm{C}_{4} \mathrm{H}_{5} \mathrm{CdO}_{4} .2 \mathrm{H}_{2} \mathrm{O}\right.$, purity $\left.~ 98.0 \%\right)$ and oleic acid were purchased from Sinopharm Chemicals reagents. Co.Ltd. Graphene powder is purchased from alfa nano Inc. Shangai, P.R.China.

\subsection{Preparation of graphene solution}


For typical preparation of graphene solution, $5 \mathrm{mg}$ of graphene powder was dissolved in $5 \mathrm{ml}$ of 1-Octadecene $(1 \mathrm{mg} / \mathrm{ml})$ and sonicated for $4 \mathrm{hr}$. The resulting solution is centrifugated for $5 \mathrm{~min}$ at $2000 \mathrm{rpm}$. The supernatant was collected and the process is repeated at 4000,6000, 8000, and 10,000 rpm to get single-layer graphene in ODE solution. The final concentration of graphene: ODE solution is $0.13 \mathrm{mg} / \mathrm{ml}$.

\subsection{In-situ synthesis of CdSe QD-Graphene}

The CdSe QDs were synthesized according to the reported literature with slight modification [28]. In a typical synthesis, the precursors are prepared in the following way. Cadmium precursor: $0.4 \mathrm{mM}$ of cadmium acetate dihydrate is taken in the round bottom flask and $10 \mathrm{ml}$ of 1-octadecene, $0.250 \mathrm{ml}$ of oleic acid, $1 \mathrm{ml}$ of graphene solution, and $1 \mathrm{ml}$ of n-octylamine is added. And then, the mixture is stirred for $30 \mathrm{~min}$ at $130{ }^{\circ} \mathrm{C}$. Selenium precursor: $2 \mathrm{mM}$ metallic selenium powder is dissolved in $6 \mathrm{ml}$ of tri-octyl phosphine (TOP) and $1.8 \mathrm{~mL}$ of toluene. For the CdSe QDsgraphene growth, $2 \mathrm{ml}$ of selenium-TOP solution was injected into the reaction flask containing cadmium precursor, and the reaction mixture was kept at $170{ }^{\circ} \mathrm{C}$. For pristine CdSe QDs growth, the procedure is followed as the same, but without graphene solution added to the cadmium precursor. The samples were taken at different time intervals of 5, 10, 15, 20, and $30 \mathrm{~min}$. The samples were purified with hexane/ methanol solution and precipitated by centrifugation at 10,000 rpm with acetone. The precipitated colloidal powder is dried and dissolved in hexane, toluene, or chloroform.

\subsection{Synthesis of CdS nanorods}

In a typical procedure, $20 \mathrm{mM}$ cadmium acetate was dissolved in $25 \mathrm{ml}$ of DI water. Then $0.250 \mathrm{ml}$ of TGA was added under vigorous stirring at room temperature. Further, sulfur source solution was prepared by dissolving $40 \mathrm{mM}$ sodium sulfide dihydrate in $50 \mathrm{ml}$ of DI water and 
added with cadmium source solution. The resulting mixture was stirred for $30 \mathrm{~min}$ and transferred into a $100 \mathrm{ml}$ Teflon-lined stainless-steel autoclave. The autoclave was maintained at $180{ }^{\circ} \mathrm{C}$ for 8 $\mathrm{hr}$ and then cooled to room temperature naturally. After cooling to room temperature, the precipitation was washed with DI water and absolute ethanol several times to remove the excess reactants and byproduct. Finally, the sample was dried in a vaccum oven at $40{ }^{\circ} \mathrm{C}$ overnight.

\subsection{Photodetector fabrication and Characterization}

Firstly, the pre-patterned ITO glass substrate was cleaned with detergent, deionized (DI) water, isopropyl alcohol, and acetone for 15 min using ultra-sonication. Further, substrates were treated with UV ozone to make the surface hydrophilic. The as-synthesized CdS NRs solution (10mg ml1 in n-butanol) was drop-casted on ITO glass substrates and dried at $80{ }^{\circ} \mathrm{C}$ for $5 \mathrm{~min}$. The CdSe QDs or graphene-CdSe QDs in n-hexane $(100 \mathrm{mg} / \mathrm{ml})$ was spin-coated at $1000 \mathrm{rpm}$ for $6 \mathrm{~s}$ and $3000 \mathrm{rpm}$ for $60 \mathrm{~s}$ and then samples were treated with ethanol washing twice. Finally, a vacuum evaporated Ag electrode $(80 \mathrm{~nm})$ was deposited to complete the device fabrication.

The UV-visible absorption spectra of as-synthesized CdSe QDs and graphene:CdSe QDs samples were analyzed on UV/vis/NIR spectrophotometer (Shimadzu, UV-3600). The steady-state photoluminescence (PL) spectra were acquired by using a fluorescence spectrophotometer (Hitachi, F-4600). Raman spectra of samples were taken at room temperature using $514.5 \mathrm{~nm}$ incident photons from an Ar ion laser (JY LabRam) in a backscattering geometry. The surface morphology of samples were acquired by a ZEISS sigma 500 field emission scanning electron

microscopy (SEM) at 30kV. A Philips CM200 tunneling electron microscope (TEM) operating at an accelerating voltage of $200 \mathrm{kV}$, with a Bruker SDD EDX system was used for transmission electron microscopy studies. The FTIR spectra of the samples were recored in a NICOLET 10 

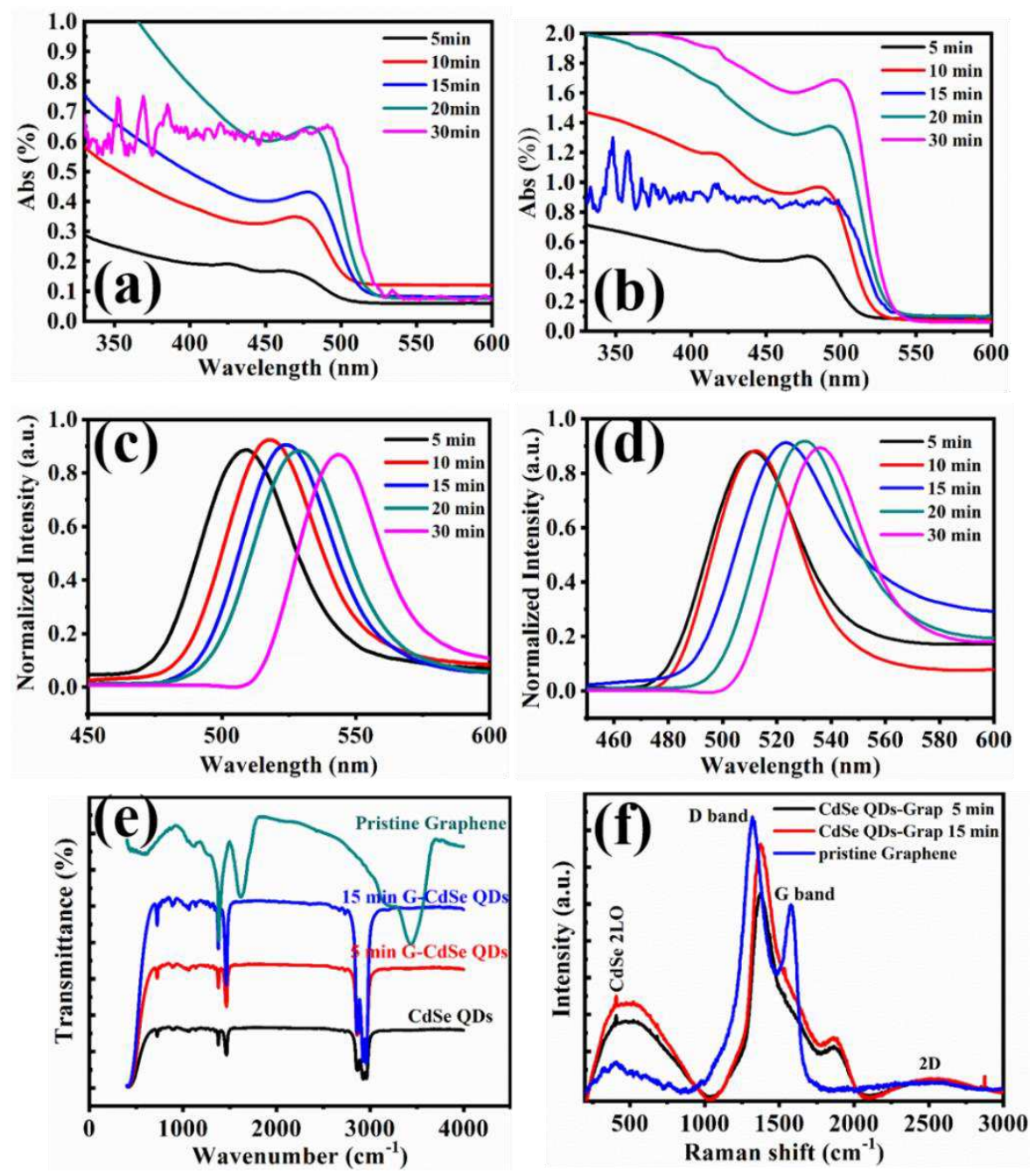

Figure.1 UV-visible absorption spectra spectra of (a) CdSe QDs (b) graphene:CdSe QDs.

Photoluminescence spectra of (c) CdSe QDs (d) graphene:CdSe QDs. (e) FTIR spectra of pure CdSe QDs, graphene: CdSe QDs synthesized for 5 min, graphene: CdSe QDs synthesized for 15 min, and pure graphene. (f) Raman spectra of the pristine graphene, graphene: CdSe QDs synthesized for $5 \mathrm{~min}$, and graphene: CdSe QDs synthesized for $15 \mathrm{~min}$.

spectrometer operating in the range of 4000 to $400 \mathrm{~cm}^{-1}$. All the electrical parameters of devices were measured by the Keithley 4200-SCS semiconductor characterization system assisted with a probe station. The irradiation was generated from monochromatic light-emitting diodes $(530 \mathrm{~nm})$, while the power of the incident radiation was tuned and measured with a power meter (Sanwa 
Mobiken LASER POWER METER LP1). All the measurements were done in air at room temperature.

\section{Results and Discussion}

The as-synthesized CdSe QDs and graphene: CdSe QDs diluted in hexane solution under UV illumination are shown in Figure S1(Supporting Information). Photographic image of the CdSe QDs and graphene: CdSe QDs nanocomposites synthesized with different reaction times under UV light irradiation at $365 \mathrm{~nm}$ shows that the samples emit different colored light indicative of the size-tunable formation of QDs with time variable. Figure 1(a) presents the UV-Vis absorption spectra of pristine CdSe QDs and graphene: CdSe QDs nanocomposite, which clearly showed the excitonic absorption edge was red-shifted as reaction time prolongs. For CdSe QDs synthesized for 5 min would show the absorption peak at $468 \mathrm{~nm}$. Subsequently, the absorption peaks were shifted to $493 \mathrm{~nm}$ for the sample synthesized for $30 \mathrm{~min}$. Compared with bulk CdSe, blue shifting of peaks indicated the emergence of the quantum confinement effect. However, the absorption spectra of graphene: CdSe QDs as in Figure1(b) showed a slight red shifting compared to pure CdSe QDs. It should be noted that graphene had no obvious absorption characteristics in the visible region; the visible light absorption was due to the contribution of CdSe QDs. The absorption spectra were changed from $479 \mathrm{~nm}$ to $498 \mathrm{~nm}$ for samples of $5 \mathrm{~min}$ to $30 \mathrm{~min}$ respectively.

The photoluminescence spectra of the pure CdSe QDs and graphene: CdSe QDs nanocomposites were displayed in Figure 1(c,d). As shown in Figure1(c), an obvious emission peak at $510 \mathrm{~nm}$ was observed for pure CdSe QDs synthesized for 5 min and it shifts towards a higher wavelength as the synthesis time prolongs. At most, CdSe QDs synthesized for 30 min would show the emission peak at $535 \mathrm{~nm}$. It could be understood that the shifts might be related to the quantum confinement effect as the size of QD evolves. However, for graphene: CdSe QDs 
nanocomposite, the emission peaks were observed at 512, 518, 526,533, and $543 \mathrm{~nm}$ for 5, 10, 15, 20- and 30-min samples respectively as shown in Table.S2 (SI). Since there is no contribution of size of QDs to shifting of emission peak, we believe that this shifting would come from photoluminescence quenching effect.

Figure 1(e) presents the FTIR spectra of CdSe QDs capped with oleic acid and graphene: CdSe QDs synthesized for $5 \mathrm{~min}$ and $15 \mathrm{~min}$. It is found that there is no peak observed at $>3000$ $\mathrm{cm}^{-1}$ as compared to TOPO capped CdSe QDs. Typical features of the Cd-Se band stretching can be observed at $724 \mathrm{~cm}^{-1}$. All other peaks are structural bonding of oleic acid with cadmium selenide QDs i.e 1378, 1466, 2861, 2873, 2931, and $2960 \mathrm{~cm}^{-1}$. The $\mathrm{CH}_{3}$ and $\mathrm{CH}_{2}$ bending deformed behavior can be observed at 1378 and $1466 \mathrm{~cm}^{-1}$ respectively. All these above observations indicated evidently that CdSe QDs were attached on the surface of graphene successfully.

Raman spectroscopy was used to characterize the ordered disordered crystal structures of carbon materials. To generalize the scheme, CdSe QDs-graphene samples synthesized for 5 min and 15 min were characterized for Raman spectra in sense of the different sizes of QDs on a graphene sheet. Raman spectra of pristine graphene sheets were also given for comparison. As indicated in Figure1(f), all three samples were exhibited the characteristic phonon modes of vibration on laser excitation. For pristine graphene sheet, two peaks at $1319.8 \mathrm{~cm}^{-1}$ and $1580.4 \mathrm{~cm}^{-}$ ${ }^{1}$ were observed and assigned to characteristic D and G bands of the two-dimensional carbon layer. Besides, a broad low-intensity peak also appears at $2500 \mathrm{~cm}^{-1}$ represents the $2 \mathrm{D}$ band of graphene. However, for the CdSe QDs functionalized graphene, an obvious characteristic peak of D and G bands are appeared along with a clear peak at $403 \mathrm{~cm}^{-1}$ which represents the overtone LO mode termed as CdSe 2LO[29]. In general, the D band at $1357 \mathrm{~cm}^{-1}$ is the breathing mode of $\pi$-point phonons of $\mathrm{A}_{1} \mathrm{~g}$ symmetry attributed to local defects and disorders, particularly the defects located 
at the edges of graphene. And also, the $\mathrm{G}$ band is assigned to the $\mathrm{E}_{2} \mathrm{~g}$ phonon of $\mathrm{sp} 2$ bonds of carbon atoms. After depositing QDs on the graphene, significant red shifting of D and G bands were observed for QD-graphene hybrids synthesized at $5 \mathrm{~min}$ and $15 \mathrm{~min}$. A redshift of D band by $55 \mathrm{~cm}^{-1}$ and $63 \mathrm{~cm}^{-1}$ were observed for $5 \mathrm{~min}$ and $15 \mathrm{~min}$ samples respectively. On the other hand, redshifts of $\mathrm{G}$ band by $277 \mathrm{~cm}^{-1}$ and $279 \mathrm{~cm}^{-1}$ were observed for 5 min and 15 min samples respectively. The G band shifting can be affected by the carrier doping levels, strain, and localized temperature[30-32]. Here, unlike the D band, the G band has shifted significantly concluded that charge transfer from QD functionalization and subsequent strain occurred by QDs loading on the graphene layer. ${ }^{[22]}$ In addition to the redshifting of characteristic peaks, the intensity ratio $\mathrm{ID}_{\mathrm{D}} / \mathrm{IG}_{\mathrm{G}}$ as shown in Figure S2(a) ( in SI) also provides the degree of QDs functionalization on graphene layers. After QDs deposition on graphene, the $\mathrm{I}_{\mathrm{D}} / \mathrm{I}_{\mathrm{G}}$ increases from 1.47 for pristine graphene to 4.69 for graphene-QDs of 15 min. Moreover, as shown in Figure S2(b) (in SI), an enhancement of $I_{2 D} / I_{G}$ ratio was observed, which suggests the presence of a QDs on the graphene layers.

Furthermore, the photo-induced kinetics of graphene: CdSe QDs are analyzed by timeresolved photoluminescence (TRPL) spectroscopy. TRPL was employed to test the emission lifetime of pure CdSe QDs (5 min), graphene: CdSe QDs (5 min), and graphene: CdSe QDs (15 min) samples using $585 \mathrm{~nm}$ excitation wavelength (see Figure $\mathbf{S 3}$ in the SI). A tri-exponential decay model was used to fit the decay curve: $(t)=A_{1} e^{-t / \tau_{1}}+A_{2} e^{-t / \tau_{2}}+A_{3} e^{-t / \tau_{3}}$, where $\mathrm{I}(\mathrm{t})$ is the time-dependent fluorescence intensity, $\mathrm{A}$ is the amplitude and $\tau$ is the lifetime. The emission lifetime of measured samples is summarized in Table S1 (in SI). It is noted that the average emission lifetime ( $\tau_{\mathrm{ave}}$ ) of graphene: CdSe QDs nanocomposites was relatively shorter than that of the corresponding pure CdSe QDs. The average lifetime of CdSe QDs is calculated to be $557.9 \mathrm{~ns}$ and graphene: CdSe QDs (5 min) is $128.34 \mathrm{~ns}$. Here, it should be considering the fact that the as- 
synthesized CdSe QDs are used for the measurement and device application without ligandexchange process. Therefore, the long-chain hydrocarbon oleic acid ligand used for the initial synthesis process form an insulating layer around each QD. Consequently, the organic ligands create an energetic barrier to charge transport[33]. However, the difference in the average lifetime between pure CdSe QDs and graphene: CdSe QDs indicates the existence of a non-radioactive pathway from the electronic interaction between quantum dots and graphene[34, 35].

The TEM and HRTEM images of representative CdSe QDs and graphene-CdSe QDs synthesized for 15 min were depicted in Figure 2. As in Figure 2 (a), CdSe QDs show a uniform size distribution with good crystalline quality. The size of the CdSe QD was in the range of $2-2.3$

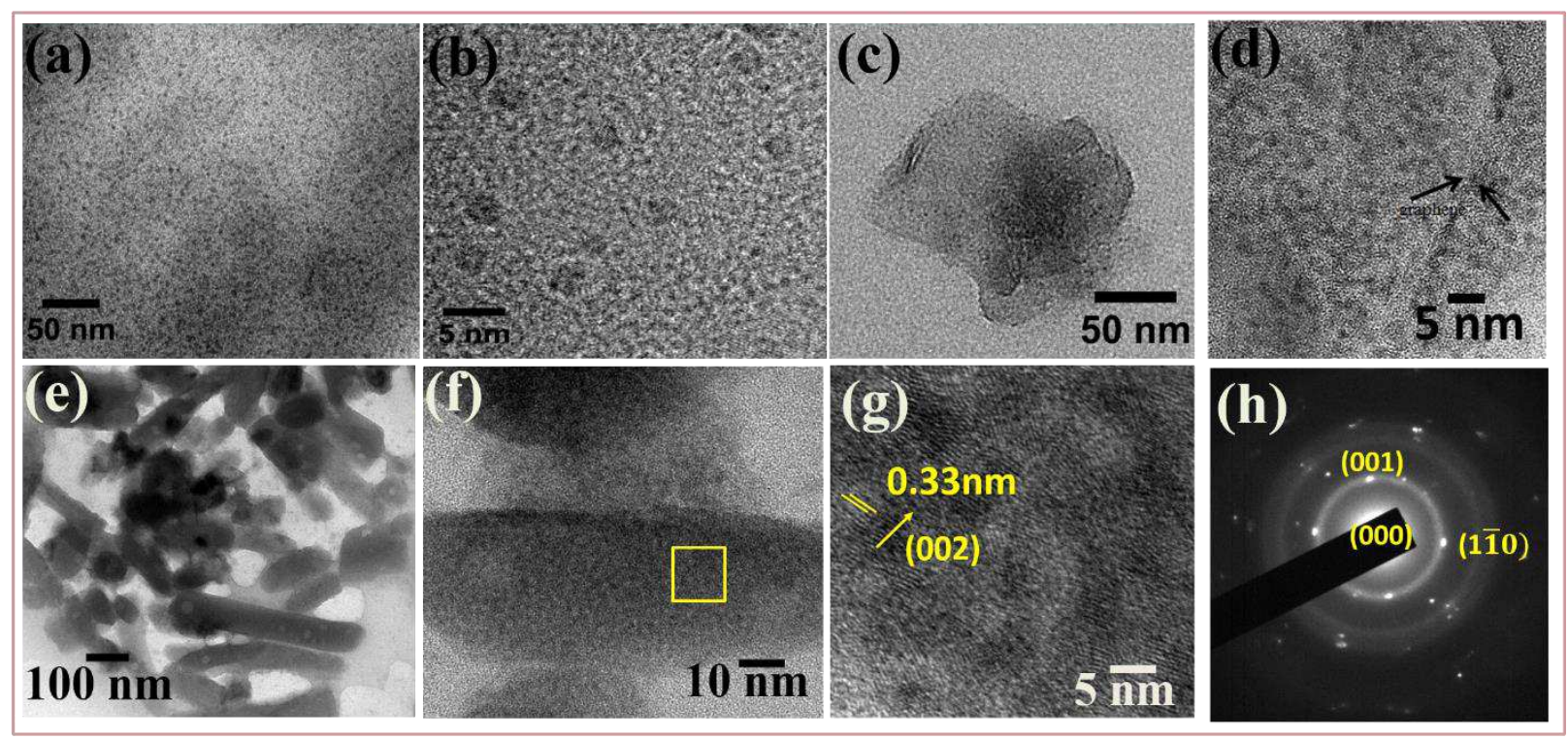

Figure.2 (a) Low magnified TEM image of CdSe QDs. (b) High magnification TEM image of CdSe QDs. (c) HRTEM image of graphene-CdSe QDs nanocomposite. (d) high magnification HRTEM image of graphene- CdSe QDs. (e) Low magnification TEM image of CdS nanorods. (f) high magnification TEM image of CdS nanorod. $(\mathrm{g})$ the enlarged portion of the selected area in CdS nanorods (yellow square in Figure2(f)). (h) selected area electron diffraction (SAED) pattern of CdS nanorods. 
$\mathrm{nm}$ as calculated from the HRTEM image for the sample synthesized for $15 \mathrm{~min}$, which is consistent with that of absorption spectra measurement. The existence of clear lattice planes on the HRTEM as in Figure 2 (b) represents the good crystallization of CdSe QDs. It is also indexed to the cubic phase of CdSe with the lattice constant of a=0.62 nm. Interestingly, synthesis of QDs in presence of graphene would show a more pronounced growth compared with pure QD synthesis. As could be seen in Figure 2(c, d), the CdSe QDs randomly attached with graphene and highly loaded on the surface of graphene. It is appeared to be clearer in shape with lattice fringes along (100) plane dominated. It seems to be the same size distribution of CdSe QDs on graphene as pure CdSe QDs. The results supported the argument that the redshifting of absorption spectra was not from the size tuning of QDs. The red shifting of absorption spectra might be from the chemical interaction of QDs on the face of graphene due to charge delocalization [36].

The phase purity and the crystal structure of the hydrothermally synthesized CdS nanorods are investigated by powder X-ray diffraction (PXRD). As shown in Figure S4 (in SI), the strong peaks indicate that the crystallinity of the product is good and no intensities of source materials or impurities are found in the spectrum. And also, the analysis of CdS data exhibits the lattice parameter of $a=4.123 \AA$ and $c=6.686 \mathrm{~A}$ which agrees with the reported literature $[37,38]$. The XRD pattern exhibits prominent, broad peaks at $2 \theta$ values of $31.5,35.2^{\circ}, 41.6^{\circ}, 50.7^{\circ}, 60.4^{\circ}$ which could be indexed to scattering from 200, 102, 102,110,112 and 104 planes respectively. The XRD shows the presence of both cubic and hexagonal phases in the synthesized CdS nanorods, which is unusual in CdS crystal growth [39]. These structural characteristics have also been supported by Raman spectra as shown in Figure S5 (in SI). As it is seen from Raman spectra, 1LO and 2LO phonon modes are observed at $298 \mathrm{~cm}^{-1}$ and $587 \mathrm{~cm}^{-1}$ respectively. In addition to the $\mathrm{LO}$ phonons and their replicas, so other peaks are also found at $101 \mathrm{~cm}^{-1}, 162 \mathrm{~cm}^{-1}, 268 \mathrm{~cm}^{-1}$, suggesting that 
the nanorods have better crystal quality.[40]. Figure 2(e) shows the TEM image of CdS nanorods, where nanorods are cylindrical with smooth surface morphology. It is noted that the average diameter of nanorod is $120 \mathrm{~nm}$ and length is $\sim 1 \mu \mathrm{m}$. The high-resolution TEM, as depicted in Figure 2(f,g), clearly indicates the single-crystalline nature of nanorods. However, the surface of nanorods consists of nanocrystals with a size of 5-6 nm, which is reflected in the SAED pattern of nanorod as shown in Figure 2(h).
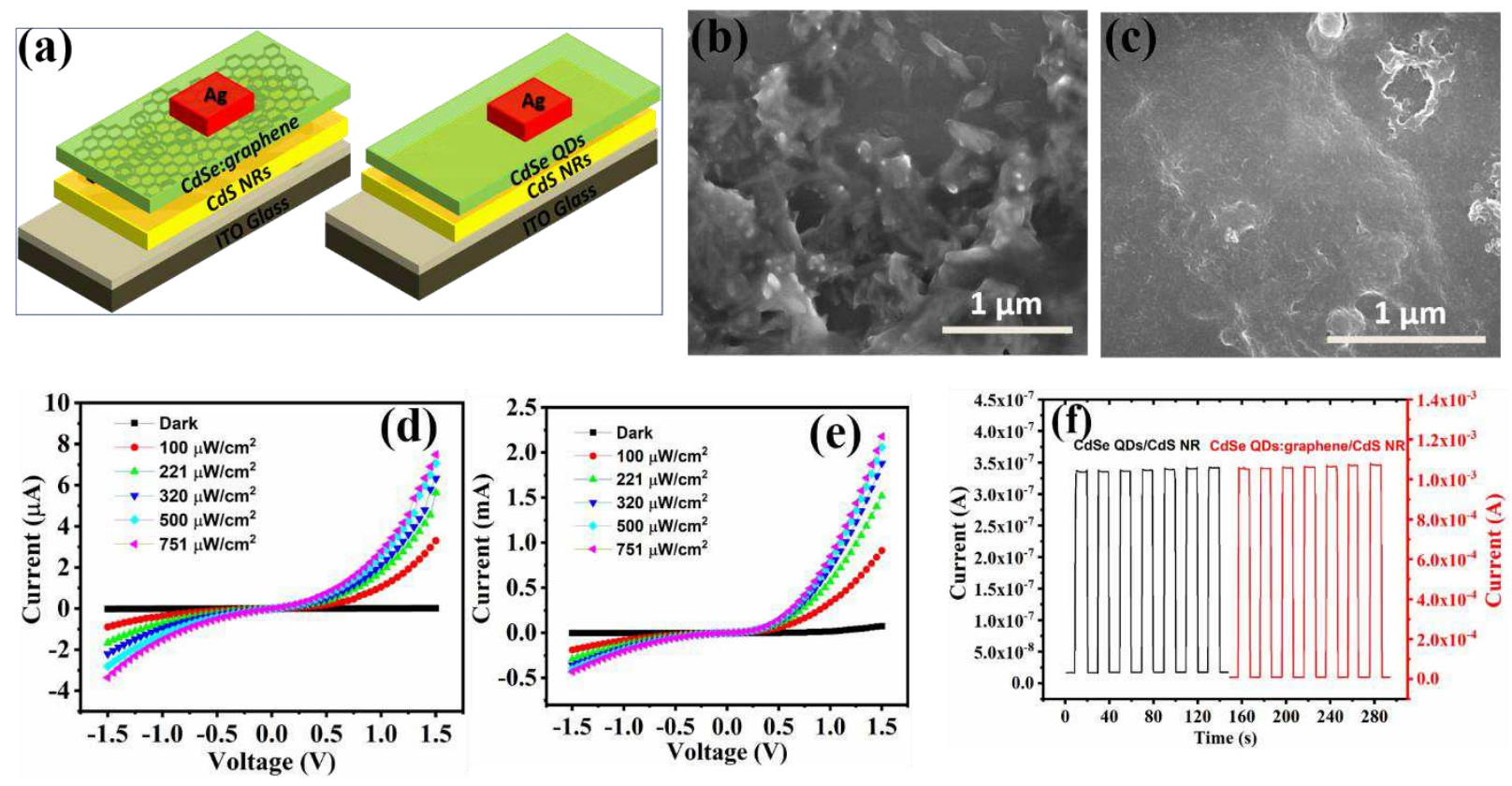

Figure.3 (a) Schematic diagram of the hybrid photodetector device structure. (b) FESEM image of CdS nanorods deposited on ITO/glass substrate. (c) CdSe QDs: graphene nanocomposite deposited on the CdS NRs/ITO/glass substrate. (d) I-V characteristics of the device with structure $\mathrm{Ag} / \mathrm{CdSe}$ QDs/CdS NRs/ITO photodetector. (e) I-V characteristics of device with structure Ag/CdSe QDs:graphene/CdS NRs/ITO photodetector. (f) The I-t curve of photodetector under bias $+1 \mathrm{~V}$. 


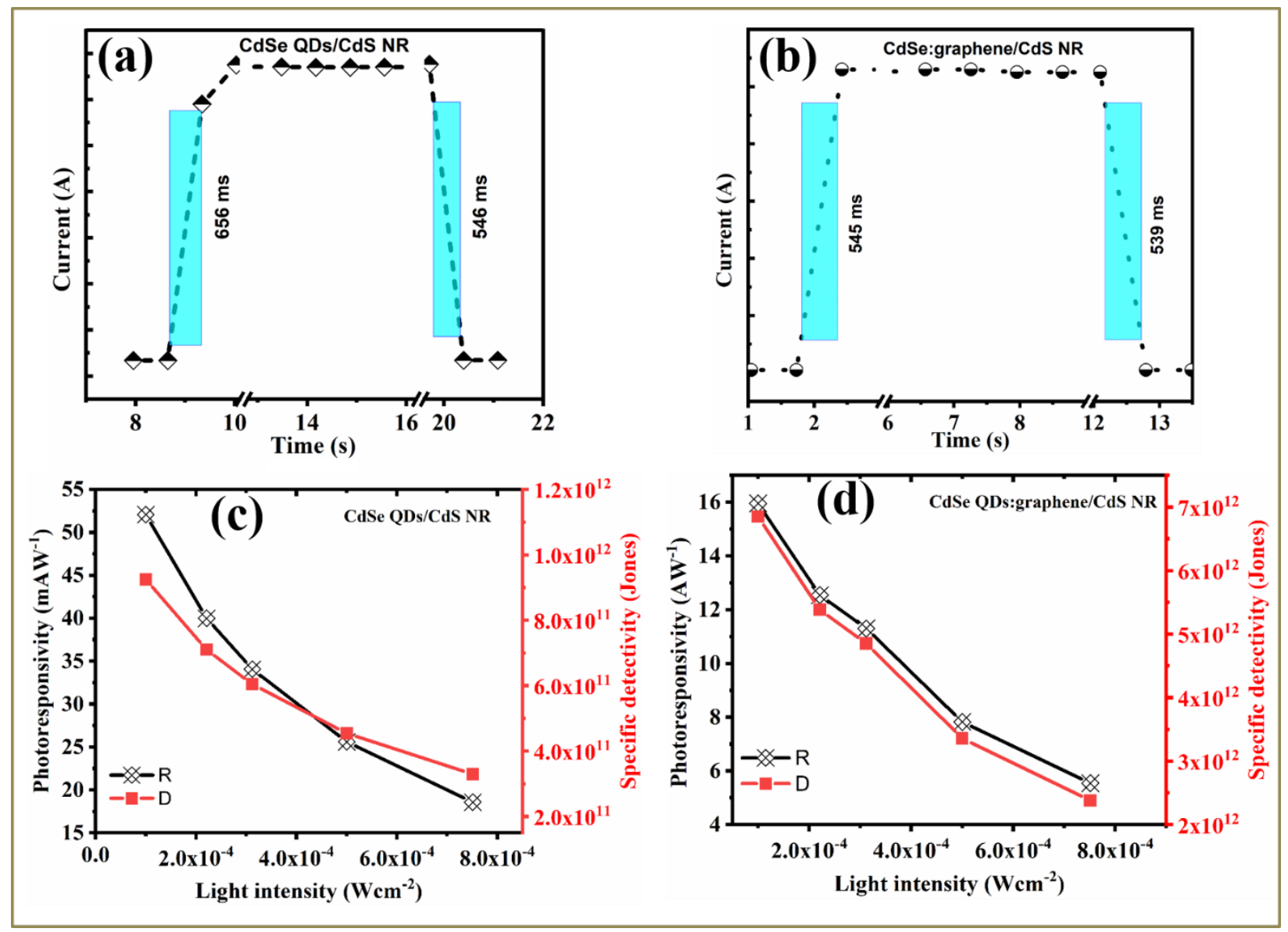

Figure.4 Temporal photoresponse of the heterojunction photodetector (a) device A. (b) device B.

(c) Photoresponsivity and specific detectivity Vs light intensity for device A. (d) Photoresponsivity and specific detectivity Vs light intensity for device B.

To demonstrate the efficient charge transfer properties of nanohybrids, a heterojunction photodetector was fabricated with device structure as shown in Figure 3(a). Two types of the device have been fabricated such as ITO/CdSe/CdS/Ag (Device A) and ITO/graphene: CdSe /CdS/Ag (Device B). The surface morphology of drop-casted CdS NRs on ITO/glass substrates is shown in Figure 3(b) and also, dense and thick distribution of CdSe QDs: graphene nanocomposite film on CdS NRs is observed as shown in Figure 3(c). The photodetector device has been characterized by applying a bias sweep from $+1.5 \mathrm{~V}$ to $-1.5 \mathrm{~V}$. Figure $3(\mathrm{~d})$ shows the I-V curves of device A under $530 \mathrm{~nm}$ laser illumination at varied power from $100 \mu \mathrm{W} / \mathrm{cm}^{2}$ to $751 \mu \mathrm{W} / \mathrm{cm}^{2}$. 
A clear rise of the photocurrent with increasing intensity of light was observed, indicating effective conversion of photon flux to photogenerated carriers. Moreover, the curve shows slight non-linear and asymmetrical behavior, confirming the proper formation of heterojunction. However, it was found that device B shows more non-linear characteristics compared to device A. It is noted that the dark current of device A and device B are $1.695 \times 10^{-8} \mathrm{~A}$ and $9.91 \times 10^{-6} \mathrm{~A}$ respectively. It clearly indicated that the dark current has been increased with graphene addition due to the increment of conductivity. At the same time, photocurrent was also increased tremendously with graphene doping from $1.0515 \mathrm{~mA}\left(100 \mu \mathrm{W} / \mathrm{cm}^{2}\right)$ to $2.799 \mathrm{~mA}\left(751 \mu \mathrm{W} / \mathrm{cm}^{2}\right)$. Moreover, the On-OFF switching of the device under $+1 \mathrm{~V}$ bias is shown in Figure3(e), displaying stable and repetitive cycles illuminated with $530 \mathrm{~nm}$ and $100 \mu \mathrm{W} / \mathrm{cm}^{2}$ light intensity, which demonstrating photodetection reversibility.

Another important characteristic of the photodetector is the linearity of photocurrent upon illumination light intensity. To examine such linearity relationship, $530 \mathrm{~nm}$ LED has been chosen with power variation from $100 \mu \mathrm{W} / \mathrm{cm}^{2}$ to $751 \mu \mathrm{W} / \mathrm{cm}^{2}$ at bias $+1 \mathrm{~V}$ as shown in Figure S6 (in SI). Fitting the plot with the power-law equation as $\mathrm{I}_{\mathrm{ph}}=\mathrm{AP}^{\alpha}$, the value of $\alpha$ is 0.43 and 0.39 for device A and device B, respectively. The obvious deviation from the ideal value of 1 is attributed to the loss of photoexcited carriers through recombination. Both defects and impurities may act as charge recombination centers, which could be filled by photoexcited carriers as the light intensity increases. Moreover, the slight increase of $\alpha$ value indicates the inherent role of graphene as the electron collector and subsequent transportation.

Photoresponse time plays an important role in photodetector behavior. Figure4(a,b) shows the rise and decay time upon $\mathrm{V}_{\text {bias }}=+1 \mathrm{~V}$ for device $\mathrm{A}$ and device $\mathrm{B}$ under $530 \mathrm{~nm}$ illumination and 100 $\mu \mathrm{Wcm}^{-2}$ light intensity. The rise time is defined as the time gap between $10 \%$ of the "off" state to 
$90 \%$ of the "on" state and decay time is defined as the opposite. The decay time shows a slight decrement from $546 \mathrm{~ms}$ to $539 \mathrm{~ms}$, while the rise time decreased from $656 \mathrm{~ms}$ to $545 \mathrm{~ms}$ after introducing graphene with CdSe QDs. Considering the fact that charge mobility in quantum dot films is limited by grain boundaries, the presence of graphene sheets provides an additional

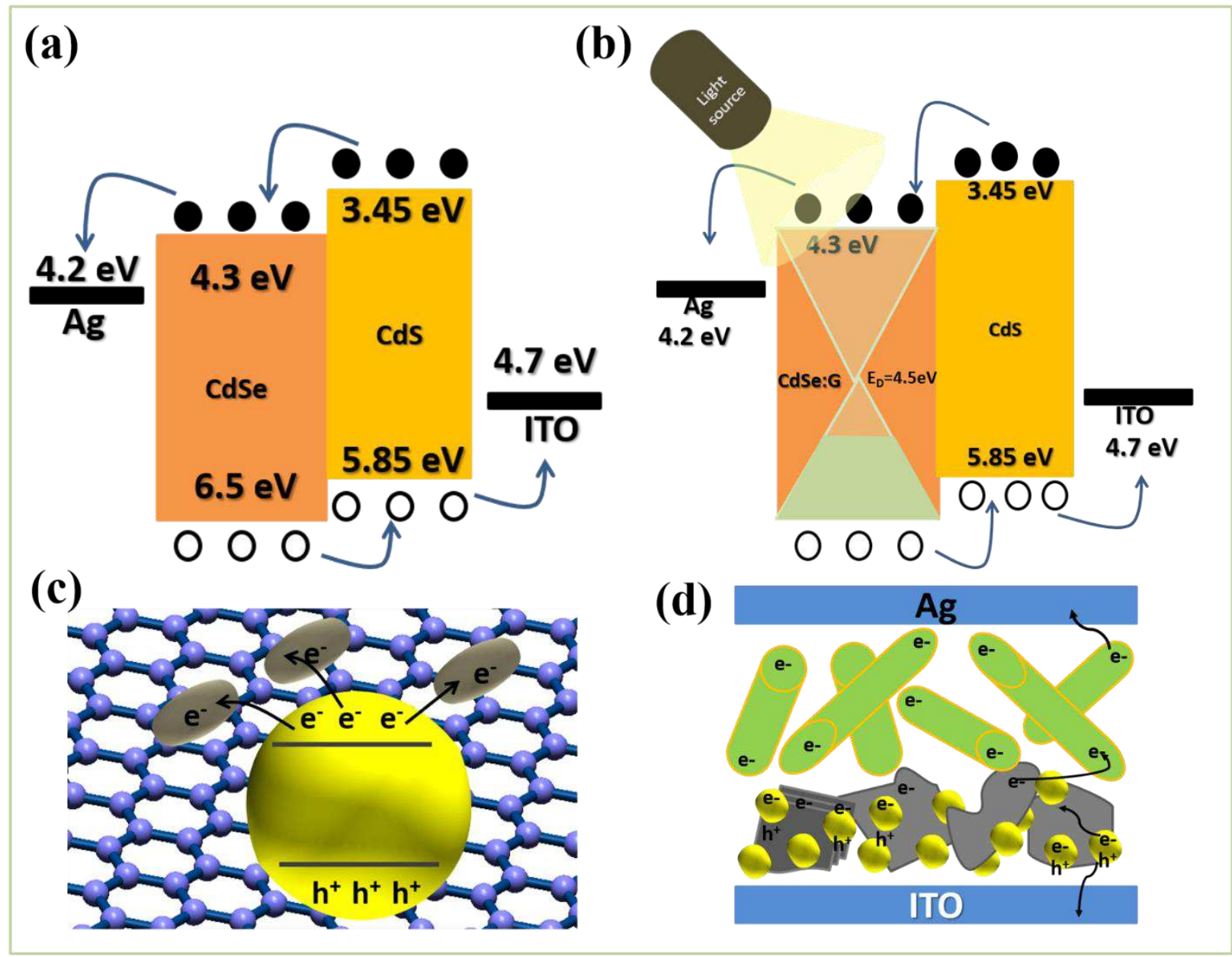

Figure.5 Schematic representation of charge carrier dynamics in (a) CdSe QDs/CdS NRs (device A) (b)CdSe QDs:Gr/CdS NRs (device B) (c) Excited state interaction between CdSe QDs and graphene. (d) shematics of charge carrier transportation in CdSe QDs:Gr/CdS NRs heterojunction photodetector.

conducting channel for charge transport[41, 42]. Therefore, device B shows better performance rather than device $\mathrm{A}$. 
Furthermore, the performance of photodetectors is evaluated in terms of key parameters such as photoresponsivity (R), Specific detectivity (D*), external quantum efficiency (EQE). The Photoresponsivity $R$ is expressed as $R=I_{p} P S$, where $I_{p}=I_{\text {light }}-I_{d a r k}, P$ is the incident power density and $\mathrm{S}$ is the effective device area. $\mathrm{D}^{*}$ is defined in terms of responsivity $\mathrm{R}$ and its simplified form

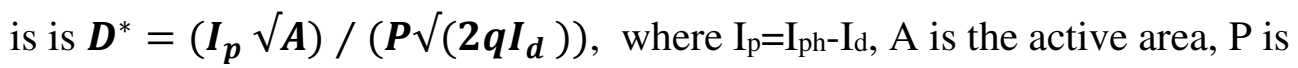

the incident power density, $\mathrm{q}$ is the coulombic charge. As shown in Figure $4 \mathrm{c}$, the maximum photoresponsivity and specific detectivity for device $\mathrm{A}$ is calculated to be $18.57 \mathrm{mAW}^{-1}$ and $9.24 \times 10^{11}$ Jones respectively. On the other hand, device B shows an enhanced photoresponsivity and specific detectivity of $15.95 \mathrm{AW}^{-1}$ and $6.85 \times 10^{12}$ Jones respectively as depicted in Figure 4(d). Moreover, incident power-dependent $\mathrm{R}$ and $\mathrm{D}^{*}$ indicate that $\mathrm{R}$ and $\mathrm{D} *$ have shown higher value at a weak light signal. Also, both $\mathrm{R}$ and $\mathrm{D}^{*}$ decreased gradually with increasing the light intensity for both device A and device B. Therefore, the results strongly manifest the existence of considerable recombination loss of charge carriers in the device. This behavior has also been observed in other systems such as $\mathrm{Gr} / \mathrm{PbS}$, perovskite nanostructures, etc.[19, 43, 44].

Figure 5(a,b) shows a schematic diagram of the energy bands corresponding to the carrier transport mechanisms of the holes and electrons during light illumination for device A and device B. When a $530 \mathrm{~nm}$ light illuminates the photodetectors through the top electrode, the photons penetrate the CdSe QDs layer, resulting in the creation of the excitons. Because of the band alignment and potential difference between the band positions, the photo-generated electrons follow the heterojunction mechanism, where the electrons present in the CB of CdS NRs layer are transferred to the CB of CdSe QDs layer and to the Ag electrode and holes in the VB of CdSe QDs are transferred to VB of CdS NRs and to the ITO (Figure 5(a))[45]. On the other hand, in device B, 
Table.1 Summary of key device performance parameters of this work and other reported graphene-QD based devices.

\begin{tabular}{|c|c|c|c|c|c|}
\hline Device structure & $\begin{array}{c}\text { Wavelength } \\
(\mathrm{nm})\end{array}$ & $\begin{array}{c}\text { Photoresponsivity } \\
\mathrm{R} \\
\left(\mathrm{AW}^{-1}\right)\end{array}$ & $\begin{array}{c}\text { Specific } \\
\text { detectivity D* } \\
(\text { Jones })\end{array}$ & $\begin{array}{c}\text { Rise/decay time } \\
(\mathrm{ms})\end{array}$ & Ref. \\
\hline Graphene/PbSe/TiO2 & 350 & 0.506 & $3 \times 10^{13}$ & $0.003 / 0.053$ & 46 \\
\hline $\begin{array}{c}\text { Ge QDs:RGO/ZnO } \\
\text { PET/graphene/CdS/Au }\end{array}$ & 1400 & 9.7 & $7.98 \times 10^{12}$ & $0.004 / 0.009$ & 47 \\
\hline $\begin{array}{c}\text { ZnO } \\
\text { NWs/graphene/CdS }\end{array}$ & 450 & 40 & - & - & 49 \\
\hline $\begin{array}{c}\text { Graphene flake/ZnO } \\
\text { nanotube }\end{array}$ & 365 & 0.043 & - & $5 / 5$ & 50 \\
\hline $\begin{array}{c}\text { InGaAs/Graphene } \\
\text { CsPbBr3/Graphene }\end{array}$ & 1550 & 7.6 & - & $68000 / 58000$ & 51 \\
\hline $\begin{array}{c}\text { Ag/CdSe QDs /CdS } \\
\text { NRs/ITO }\end{array}$ & 530 & 0.052 & $9.24 \times 10^{11}$ & $656 / 546$ & This work \\
\hline $\begin{array}{c}\text { Ag/graphene:CdSe } \\
\text { QD/CdS NRs/ITO }\end{array}$ & 530 & 15.95 & $6.85 \times 10^{12}$ & $545 / 539$ & This work \\
\hline
\end{tabular}

charge excitons are generated in the CdSe QDs: graphene nanocomposite under light illumination and charge carrier separation is occurred under applied electric field, however, in presence of graphene, charge carrier separation is enhanced due to nano heterojunction at the interface of CdSe QDs/graphene (Figure 5(c)). Besides, a higher conductive channel provided by graphene can induce the charge carrier transportation effectively. Therefore, the electrons and holes are accumulated at the Ag and the ITO layers, respectively, resulting in the generation of the photocurrent in the photodetectors (Figure.5 (d)). It is believed that the photodetector performance could be further improved by the proper ligand-exchange process to overcome the resistance provided by oleic acid and designing of the device structure. We also compared the performance of our device with other graphene nanocomposite-based photodetectors as shown in Table 1.

\section{Conclusion}

In summary, we have successfully synthesized the graphene: CdSe QDs nanocomposite at a low-temperature regime by a one-pot solvothermal method and subsequently demonstrated the heterojunction photodetector. It is found that the synergistic interaction between CdSe QDs and graphene facilitating the separation of electron-hole pairs and prolong the lifetime of the charge 
carriers. Interestingly, the fabrication of heterojunction photodetector Ag/graphene: $\mathrm{CdSe}$ QDs/CdS NRs/ITO exhibits higher photoresponsivity and detectivity up to $15.95 \mathrm{AW}^{-1}$ and $6.85 \times 10^{12}$ Jones respectively. The appropriate structure and band alighnment in graphene:CdSe QDs/CdS NR heterojunction benefits the visible light absorption and enhanced charge transfer. The present study reveals that the formation of nano junction by introducing graphene into QD layers is a good strategy to improve the charge carrier separation and transportation that results in the enhancement of the photosensing capability of the photodetector.

\section{Acknowledgments}

This work was supported by the National Key Research and Development Program of China (Grant No. 2018YFB2200500), the National Natural Science Foundation of China (Grant no. 61974170,61934007,61675195), the Opened Fund of the State Key Laboratory of Integrated Optoelectronics No. IOSKL2018KF17, the Beijing Municipal Science and Technology Commission Project (Grant No. Z191100004819011).

\section{Conflict of Interest}

The authors declare no conflict of interest.

\section{References}

[1] Bonaccorso F, Sun Z, Hasan T and Ferrari A C 2010 Nat.Photonics., 4 611-22

[2] Reddy D, Register L F, Carpenter G D and Banerjee S K 2011 J.Phys D.Appl.Phys., 44313001

[3] Freitag M, Low T, Xia F and Avouris P 2013 Nat.Photonics., 7 53-9

[4] Liu J-Y, Li X-X, Huang J-R, Li J-J, Zhou P, Liu J-H and Huang X-J 2017 J.Mater.Chem A., 5 5977-94 
[5] Danielson E, Sontakke V A, Porkovich A J, Wang Z, Kumar P, Ziadi Z, Yokobayashi Y and Sowwan M 2020 Sens Actuators B Chem., 320128432

[6] Akbari-Sharbaf A, Ezugwu S, Ahmed M S, Cottam M G and Fanchini G 2015 Carbon, 95 199-207

[7] Khan M, Tahir M N, Adil S F, Khan H U, Siddiqui M R H, Al-warthan A A and Tremel W 2015 J.Mater.Chem A., 3 18753-808

[8] Song X, Zhang Y, Zhang H, Yu Y, Cao M, Che Y, Dai H, Yang J, Ding X and Yao J 2017 Nanotechnol., 28145201

[9] Murray C B, Norris D J and Bawendi M G 1993 J.Am.Chem.Soc., 115 8706-15

[10] Pu Y, Cai F, Wang D, Wang J-X and Chen J-F 2018 Ind.Eng.Chem.Res., 57 1790802

[11] Wu J, Chen S, Seeds A and Liu H 2015 J.Phys D.Appl.Phys., 48363001

[12] Li J and Zhu J-J 2013 Analyst 138 2506-2515

[13] Zhao N, Osedach T P, Chang L-Y, Geyer S M, Wanger D, Binda M T, Arango A C, Bawendi M G and Bulovic V 2010 ACS Nano 4 3743-3752

[14] Tong L, Qiu F, Zeng T, Long J, Yang J, Wang R, Zhang J, Wang C, Sun T and Yang Y 2017 RSC Adv., 7 47999-8018

[15] Guo C X, Yang H B, Sheng Z M, Lu Z S, Song Q L and Li C M 2010 Angew.Chem.Int., 49 3014-3017

[16] Goossens S, Navickaite G, Monasterio C, Gupta S, Piqueras J J, Pérez R, Burwell G, Nikitskiy I, Lasanta T, Galán T, Puma E, Centeno A, Pesquera A, Zurutuza A, Konstantatos G and Koppens F 2017 Nat.Photonics., 11 366-371 
[17] Geng X, Niu L, Xing Z, Song R, Liu G, Sun M, Cheng G, Zhong H, Liu Z, Zhang Z, Sun L, Xu H, Lu L and Liu L 2010 Adv.Mater., 22 638-42

[18] Kim Y-T, Han J H, Hong B H and Kwon Y-U 2010 Adv.Mater., 22 515-518

[19] Sun Z, Liu Z, Li J, Tai G-a, Lau S-P and Yan F 2012 Adv.Mater., 24 5878-5883

[20] Chen W, Ahn S, Balingit M, Wang J, Lockett M and Vazquez-Mena O 2020 Nanoscale., 12 4909-4915

[21] Cook B, Gong M, Corbin A, Ewing D, Tramble A and Wu J 2019 ACS Omega 4 22497-22503

[22] Zhang B, Wang K, Chang R, Yi X, Zhang Y and Wang S 2019 J.Phys.Chem C., $12324943-24948$

[23] Zedan A F, Sappal S, Moussa S and El-Shall M S 2010 J.Phys.Chem C., 114 1992019927

[24] Miao X, Gosztola D J, Sumant A V and Grebel H 2018 Nanoscale., 10 7040-6

[25] Cao S, Wang J, Ma F and Sun M 2018 Nanotechnol., 29145202

[26] Cao A, Liu Z, Chu S, Wu M, Ye Z, Cai Z, Chang Y, Wang S, Gong Q and Liu Y 2010 Adv.Mater., 22 103-6

[27] Konstantatos G, Badioli M, Gaudreau L, Osmond J, Bernechea M, de Arquer F P G, Gatti F and Koppens F H L 2012 Nat.Nanotechnol., 7 363-8

[28] Siy J T, Brauser E H, Thompson T K and Bartl M H 2014 J.Mater.Chem C., 2 67582

[29] Yükselici M H, Aşıkoğlu Bozkurt A and Ömür B C 2013 Mater. Res.Bull., 48 2442- 
[30] Yoon D, Moon H, Son Y-W, Choi J S, Park B H, Cha Y H, Kim Y D and Cheong H 2009 Phys.Rev B 80125422

[31] Ni Z H, Yu T, Lu Y H, Wang Y Y, Feng Y P and Shen Z X 2009 ACS Nano 3 483-

[32] Calizo I, Balandin A A, Bao W, Miao F and Lau C N 2007 Nano Lett., 7 2645-2649

[33] Ren Z, Yu J, Pan Z, Wang J and Zhong X 2017 ACS Appl.Mater.Interfaces., 9 $18936-44$

[34] Jung M-H and Chu M-J 2014 Nanoscale., 6 9241-9

[35] Tang X, Zu Z, Zang Z, Hu Z, Hu W, Yao Z, Chen W, Li S, Han S and Zhou M 2017 Sens Actuators B Chem., 245 435-40

[36] Zhang H, Lv X, Li Y, Wang Y and Li J 2010 ACS Nano 4 380-6

[37] Yan P, Xie Y, Qian Y and Liu X 1999 Chem.Commun., 1293-4

[38] Yang J, Zeng J-H, Yu S-H, Yang L, Zhou G-e and Qian Y-t 2000 Chem.Mater., 12 $3259-63$

[39] Ascencio J A, Santiago P, Rendón L and Pal U 2004 Appl.Phys.A., 78 5-7

[40] Hu C, Zeng X, Cui J, Chen H and Lu J 2013 J.Phys.Chem C., 117 20998-1005

[41] Koleilat G I, Levina L, Shukla H, Myrskog S H, Hinds S, Pattantyus-Abraham A G and Sargent E H 2008 ACS Nano 2 833-40

[42] Kamat P V 2010 J.Phys.Chem.Lett., 1 520-7

[43] Ahn S, Chen W, Moreno-Gonzalez M A, Lockett M, Wang J and Vazquez-Mena O 2020 Adv.Elect.Mater., 62000014

[44] Chandrasekar P V, Yang S, Hu J, Sulaman M, Shi Y, Saleem M I, Tang Y, Jiang Y and Zou B 2019 Nanoscale., 11 5188-96 
[45] Skromme B J and Sujan G K 2018 Reference Module in Materials Science and Materials Engineering: Elsevier.

[46] Manga K K, Wang J, Lin M, Zhang J, Nesladek M, Nalla V, Ji W and Loh K P 2012 Adv.Mater., 24 1697-702

[47] Liu X, Ji X, Liu M, Liu N, Tao Z, Dai Q, Wei L, Li C, Zhang X and Wang B 2015 ACS Appl.Mater.Interfaces., 7 2452-8

[48] Chan Y, Dahua Z, Jun Y, Linlong T, Chongqian L and Jun S 2020 Physica E Low Dimens.Systs.Nanostruct., 124114216

[49] Huang G, Zhang P and Bai Z 2019 J.Alloys.Compd., 776 346-52

[50] Huang B-R, Saravanan A and Lu H-C 2020 Adv.Mater.Interfaces., 71901694

[51] Yang Q, Wu Q, Luo W, Yao W, Yan S and Shen J 2019 Mater.Res.Express., 6 116208

[52] Che Y, Cao X, Zhang Y and Yao J 2021 J.Mater.Sci., 56 2341-6 

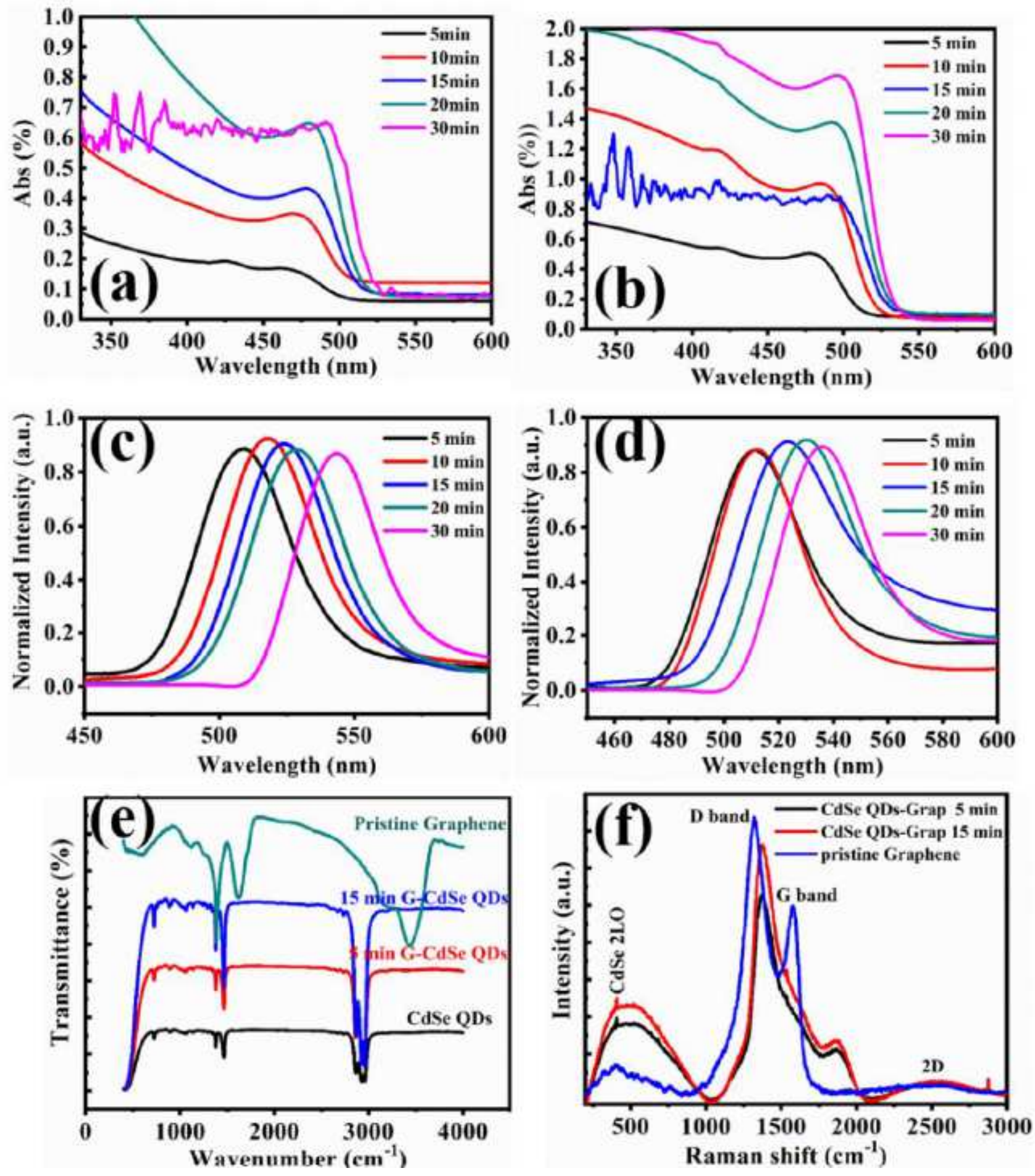

Figure 1

UV-visible absorption spectra spectra of (a) CdSe QDs (b) graphene:CdSe QDs. Photoluminescence spectra of (c) CdSe QDs (d) graphene:CdSe QDs. (e) FTIR spectra of pure CdSe QDs, graphene: CdSe QDs synthesized for 5 min, graphene: CdSe QDs synthesized for 15 min, and pure graphene. (f) Raman 
spectra of the pristine graphene, graphene: CdSe QDs synthesized for 5 min, and graphene: CdSe QDs synthesized for $15 \mathrm{~min}$.

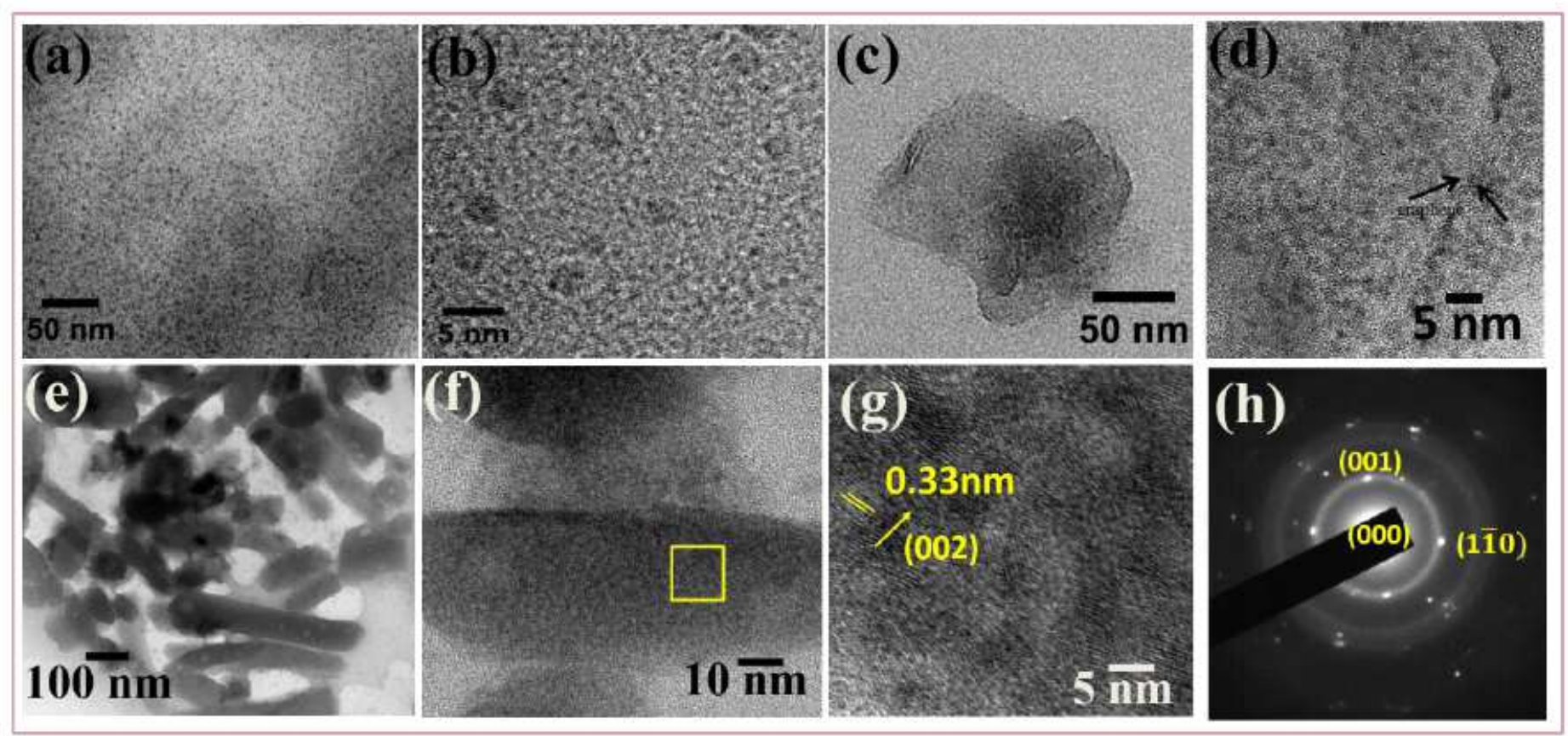

Figure 2

(a) Low magnified TEM image of CdSe QDs. (b) High magnification TEM image of CdSe QDs. (c) HRTEM image of graphene-CdSe QDs nanocomposite. (d) high magnification HRTEM image of graphene- CdSe QDs. (e) Low magnification TEM image of CdS nanorods. (f) high magnification TEM image of CdS nanorod. $(\mathrm{g})$ the enlarged portion of the selected area in CdS nanorods (yellow square in Figure2(f)). (h) selected area electron diffraction (SAED) pattern of CdS nanorods.
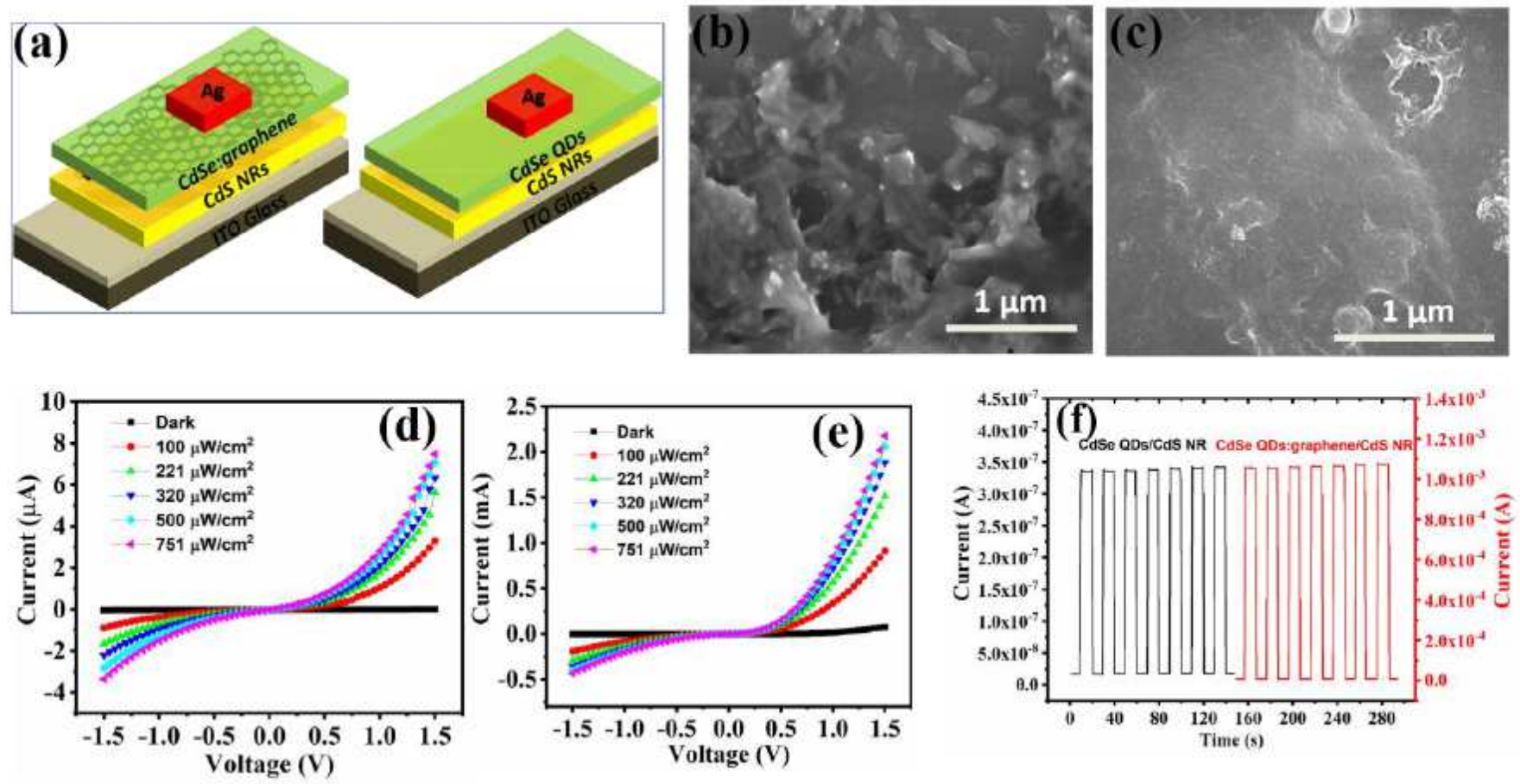
Figure 3

(a) Schematic diagram of the hybrid photodetector device structure. (b) FESEM image of CdS nanorods deposited on ITO/glass substrate. (c) CdSe QDs: graphene nanocomposite deposited on the CdS NRs/ITO/glass substrate. (d) I-V characteristics of the device with structure Ag/CdSe QDs/CdS NRs/ITO photodetector. (e) I-V characteristics of device with structure Ag/CdSe QDs:graphene/CdS NRs/ITO photodetector. $(\mathrm{f})$ The l-t curve of photodetector under bias $+1 \mathrm{~V}$.
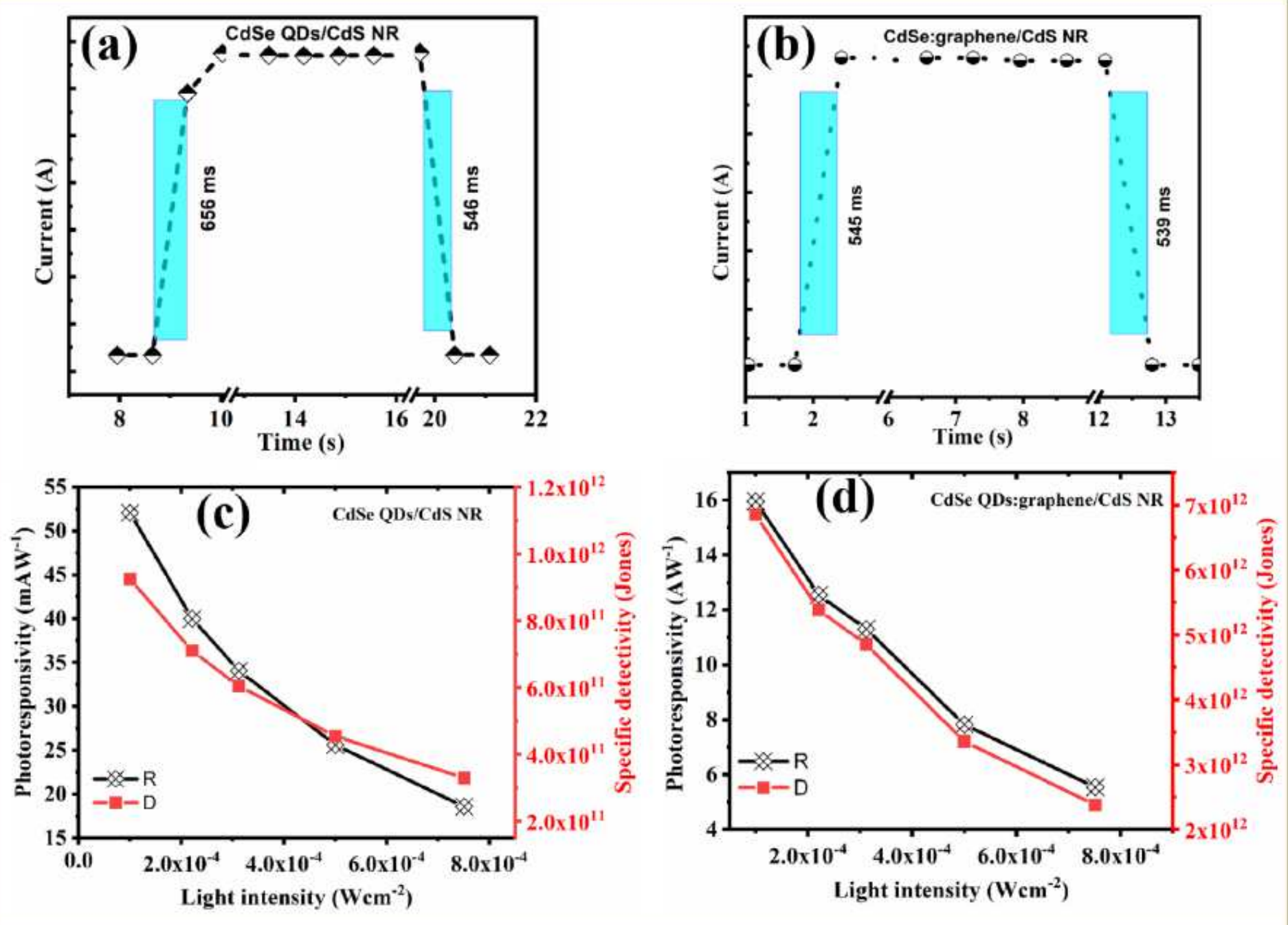

Figure 4

Temporal photoresponse of the heterojunction photodetector (a) device A. (b) device B. (c) Photoresponsivity and specific detectivity Vs light intensity for device A. (d) Photoresponsivity and specific detectivity Vs light intensity for device B. 


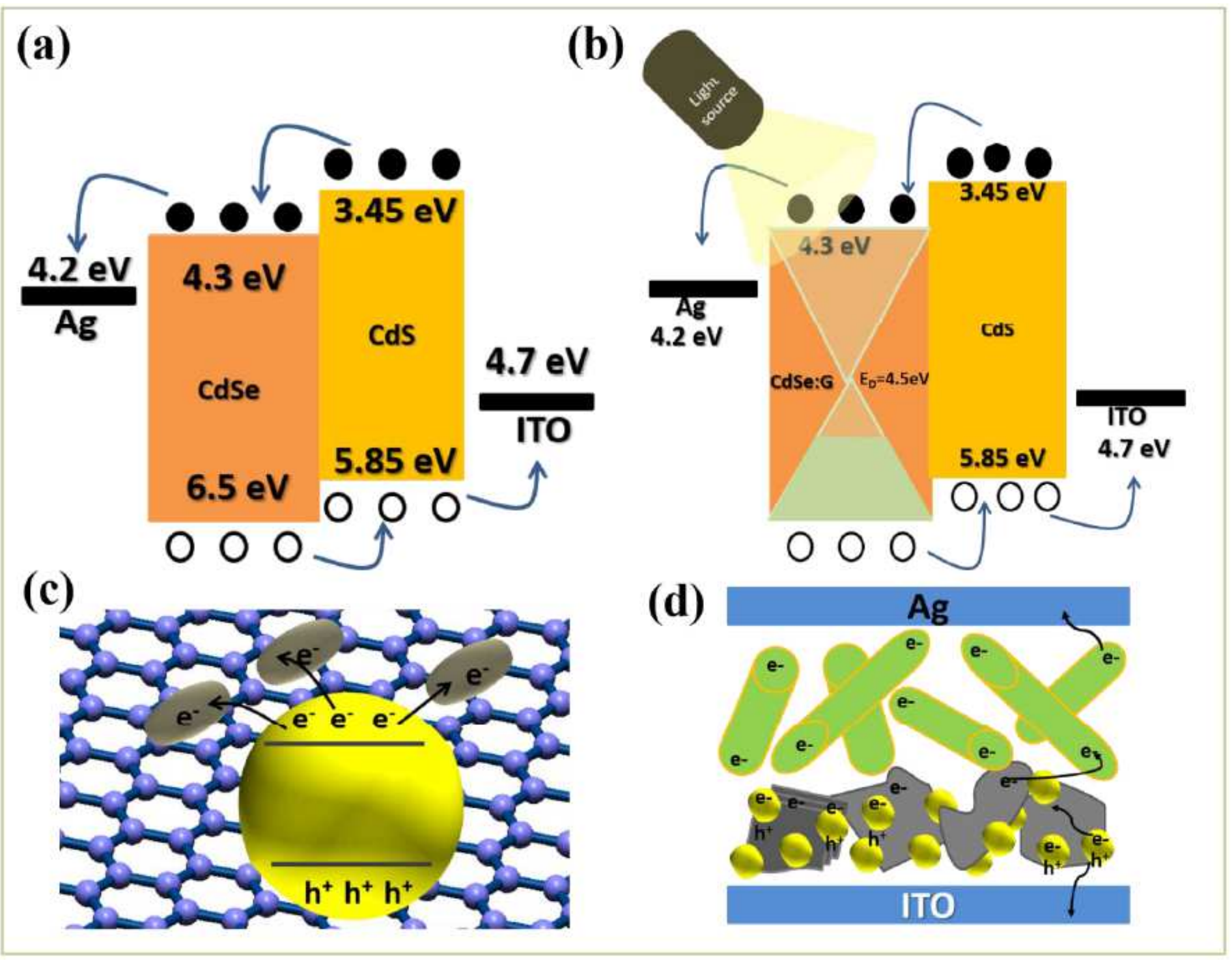

Figure 5

Schematic representation of charge carrier dynamics in (a) CdSe QDs/CdS NRs (device A) (b)CdSe QDs:Gr/CdS NRs (device B) (c) Excited state interaction between CdSe QDs and graphene. (d) shematics of charge carrier transportation in CdSe QDs:Gr/CdS NRs heterojunction photodetector.

\section{Supplementary Files}

This is a list of supplementary files associated with this preprint. Click to download.

- SupportingInformation.docx 